\title{
The impact of COVID-19 on obstetrics and gynaecology care
}

The COVID-19 pandemic is the biggest global disturbance in living memory. Much debate has focused on economic outlay to various communities or groups by governments and health services, including access to personal protective equipment (PPE), and interventions to prevent transmission. Following the outbreak in early March 2020, South Africa, along with many other countries, is now in the midst of a 'second wave' of COVID-19 infections. The virus has forced us to question the evidence for the 'scientific' advice that is given to politicians and society. Predictions of the extent of COVID-19, for example, have often been based on calculations founded on statistical modelling, and not actual trends. This has produced diverse predictions, and may have fostered mistrust and fear among clinicians as well as society at large. One issue raised by the current COVID-19 pandemic is the conflict that exists between the needs to protect health and to preserve the economy. If simply applying maximum safety was the overriding consideration for COVID-19, all populations would be living and working from their homes and segregated from one another to prevent transmission. However, the world is based on an economic system, and no individual, family, section of society, community, region or nation can survive without resources. For many, those resources are acquired in the short term, and not stored.

\section{Obstetric response to the pandemic}

Antenatal care and childbirth cannot be postponed and, unlike the case in other disciplines, care for pregnant women had to continue throughout the various levels of lockdown. The initial concern was evidence of COVID-19 transmission from mother to child, followed by concerns about the safety of breastfeeding, and ultimately the safety of mothers, neonates and healthcare workers in the maternity wards. Concerns around the mental health status of pregnant women were not often publicly addressed, and in some instances, pregnant women did not know whether to continue antenatal care or to stay at home, increasing the risk of adverse maternal and perinatal outcomes. It is not yet clear whether stillbirth rates and maternal mortality rates have increased or decreased, particularly in low- and middle-income countries, but beyond the impact of COVID-19 on pregnancy, the impact of the pandemic itself on pregnancy has threatened the progress made through the Sustainable Development Goals.

\section{Gynaecological response to the pandemic}

The global COVID-19 pandemic necessitated immediate governmental and regulatory strategies to limit the transmissibility potential of the virus. Social distancing and restricted-access interventions, including lockdown and quarantine, resulted in the restructuring and redefining of both healthcare infrastructure and professional roles. Women were only able to seek emergency medical attention. As such, accessing essential gynaecological care, including outpatient consultations for routine gynaecological evaluation, sexual and reproductive healthcare (including contraception), access to diagnostic imaging (for chronic pelvic pain, cancer staging, infertility, etc.), continued oncology treatment and, in all probability, termination of pregnancy facilities were suspended with immediate effect.
One of the major gynaecological concerns included lack of access to contraception supplies (condoms, sanitary towels and contraceptives) and services, which could potentially exacerbate an increase in unplanned pregnancies, resulting in women accessing unsafe termination of pregnancy procedures resulting in eventual morbidity and even death. Due to the massive shifting of resources, the the United Nations Population Fund (UNFPA) estimated that the coronavirus pandemic would result in $\sim 7$ million unintended pregnancies. ${ }^{[1]}$ During lockdown, the WHO recommended that couples continue their contraceptive method of choice, or commence a safe method limited to what was available without a prescription, i.e. emergency contraception, condoms, spermicides and diaphragms.

COVID-19 has disrupted medical practice, changing even the form of service delivery. In many instances, gynaecological screening (e.g. of new contraceptive users) and consultations were performed using one of several digital technological platforms such as video calls, WhatsApp and even short message services (SMS). Routine removals and reinsertions of long-acting reversible contraception (copper intrauterine contraceptive device (IUCD), Mirena intrauterine system (IUS) and subdermal implants) were postponed (unless the couple wanted to be pregnant or suffered an acute event), since the risk of pregnancy beyond the stipulated licensed duration is extremely low.

\section{COVID-19 pandemic debates}

There has been debate over the effects of SARS-CoV-2 on human physiology, and around which treatments may be effective. There has also been debate over the response of the immune system, which may in overactivity partly explain toxicity, and in underactivity may limit protection against re-infection, especially during pregnancy. ${ }^{[2]}$ Different categories of society have varying susceptibility: at-risk groups, including the elderly and those with serious medical conditions, as well as those who are overweight, have been reported to be more vulnerable to harmful effects and death, and have been contrasted with the young, in whom very few deaths have occurred.

Measures to control virus spread have been limited by the fact that the majority of carriers are asymptomatic. The ability to limit contact of identified infected persons with others has been reduced by test results being delayed, rendering segregation and contact tracing impractical and near unachievable. A lack of evidence has been at the core of COVID-19 pandemic debates, and has taught us how little we know about viruses and their behaviour.

\section{COVID-19 and women's health}

Interestingly, emerging evidence suggests a higher mortality, morbidity and severe case rate among men than women, which may be explained by sex-based hormonal immunological differences. Oestrogen (E2) may be protective owing to its ability to induce higher levels of antibodies, and activate antibodyproducing cells. The pandemic has also influenced the treatment approach to ectopic pregnancies. While surgery remains the mainstay of treatment in haemodynamically unstable patients, treatment with methotrexate requires careful consideration. 
According to the Royal College of Obstetricians and Gynaecologists, 'it is likely the detrimental effects of methotrexate in COVID-19 are minimal in well women. Methotrexate is a mildly immunosuppressive medication, but it is not thought to be a significant risk at the dose used to treat ectopic pregnancy. It is unlikely to increase vulnerability to COVID-19 and does not require home shielding after administration. ${ }^{\text {[3] }}$

Several countries witnessed the spike of other crises, e.g. genderbased violence. This was foreseeable, given that in Sierra Leone, during an Ebola pandemic, many young girls were sexually assaulted, and teenage pregnancy increased by $65 \% \cdot{ }^{[4]}$ Mounting evidence indicates a global rise in intimate partner violence while women were 'locked down' with abusive partners. Several government and private companies are currently analysing data pertaining to the extent of the violence, and considering the implementation of creative solutions such as digital reporting tools, on-demand provision of hotel rooms and training pharmacists to assist victims.

While emergency gynaecological surgery has continued, elective surgeries for women with several benign gynaecological conditions such as endometriosis, abnormal uterine bleeding and benign ovarian pathology have been postponed in COVID-19-designated hospitals. Teleconsultations, empirical over-the-counter treatments and alternative pain management strategies have been recommended prior to presenting to the emergency room.

As restrictions ease, several healthcare services have resumed at a slow to moderate capacity. Ongoing considerations for surgery include:

- pre-operative COVID-19 screening

- continued PPE attire and behaviour

- mitigating exposure to aerosol-generating procedures.
Regulatory authorities and policy-makers need to consider sustainable, effective and efficient routine healthcare pathways for continued delivery of safe obstetric and gynaecological services during the pandemic.

Zeelha Abdool, MB ChB, MMed, FCOG (SA),

MPhil (Applied Ethics), $\mathrm{PhD}$

Department of Obstetrics and Gynaecology, University of Pretoria, South Africa

zeelha.abdool@up.ac.za

Salome Maswime, MB ChB, MMed, FCOG (SA), PhD

Global Surgery Division, Department of Surgery, University of Cape Town, South Africa

salome.maswime@uct.co.za

William Edridge, MB BChir (Cantab), FCOG (SA), MRCOG (UK) Department of Obstetrics and Gynaecology, University of the Witwatersrand, Johannesburg, South Africa

william.edridge@gmail.com

S Afr J Obstet Gynaecol 2020;26(2):44-45. https://doi.org/10.7196/SAJOG.2020. v26i 2.02028

1. United Nations Population Fund. Impact of COVID-19 on family planning and ending gender-based violence, female genital mutilation and child marriage. New York: UNFPA, 2020. https://www. unfpa.org/sites/default/files/resource-pdf/COVID-19_impact_brief_for_UNFPA_24_April_2020_1. pdf (accessed 15 January 2021).

2. Chen M, Zeng J, Liu X, et al. Changes in physiology and immune system during pregnancy and coronavirus infection: A review. Eur J Obstet Gynecol Reproductive Biol 2020,255:124-128. https:// doi.org/10.1016/j.jogrb.2020.10.035

3. Ectopic Pregnancy Trust. Ectopic pregnancy and COVID-19. https://ectopic.org.uk/patients/ectopicpregnancy-covid-19-coronavirus/ (accessed 13 January 2021)

4. Onyango MA, Resnick K, Davis A, Shah RR. Gender-based violence among adolescent girls and young women: A neglected consequence of the West African Ebola outbreak. In: Schwartz DA, Anoko JN, Abramowitz, eds. Pregnant in the Time of Ebola. London: Springer Nature, 2019:121-132. 